\title{
Hypofractionated Whole Breast Radiation and Partial Breast Radiation for Early-Stage Breast Cancers: An Update on Progress
}

\author{
Beryl McCormick, MD
}

\begin{abstract}
This article provides an update of recent progress using partial breast irradiation (PBI) for the treatment of early-stage breast cancer, rather than whole breast radiotherapy (WBRT), which is the standard of care. Several large, prospective, randomized trials are nearing target accrual or have been completed, including the NSABP/RTOG trial, the Milan-based intraoperative radiation trial, and the international TARGIT trial, and the status of each is discussed. The American Society for Radiation Oncology has also published a consensus statement to guide the use of PBI until some of the phase III trials are more mature. Finally, several articles have appeared recently, reporting unexpected adverse effects of $\mathrm{PBI}$ in small series, and this information is reviewed. Several recent prospective trials of WBRT are also discussed, with the theme of comparing the standard 25 fractions to a shortened, hypofractionated trial arm delivering equivalent doses of WBRT in approximately 15 treatments, another radiation strategy for a shortened course of treatment after breastconserving surgery. (JNCCN 2012;10:1061-1164)
\end{abstract}

convened a consensus panel of experts and published guidelines for PBI use in 2009.

Perhaps as importantly, focusing on one of the goals of PBI, namely increasing the convenience of breast radiation for patients, several phase III trials have been reported comparing WBRT using the standard 25-fraction course of treatment versus a hypofractionated regimen using just 13 to 16 treatments. These large trials with long follow-up times show equivalence with both regimens, and therefore produce a much more patientfriendly treatment timeline for the delivery of WBRT. One major rationale for PBI has been the convenience to patients.

As with any new technique, several reports from major centers have been published recently showing some unanticipated side effects associated with the use of PBI.

\section{The Status of the Prospective Randomized Trials}

The NSABP B-39/RTOG 0413 trial accrued extremely well during its first years open. Briefly, the trial prospectively randomized women with early-stage breast cancer to receive either conventional WBRT or PBI, which could be delivered through either catheter or MammoSite (Hologic) brachytherapy, or with external beam PBI. The technique was to reflect the expertise of the treating oncologist, with PBI treatments scheduled twice per day for 5 days. The original target accrual was 3000 patients. However, an analysis in 2007 of the mix of patients entered into the trial showed that most of the women had very "good risk" disease, associated with a low event rate compared with the model used to plan the trial. ${ }^{2}$

Thus, this trial was modified in June 2007, and revised entry criteria were limited to women who were 
premenopausal, or had estrogen receptor (ER)-negative or node-positive disease only. The target accrual was increased to 4300 patients, and the trial is now projected to close in late 2012 or early 2013. Of interest, looking only at the women randomized to receive $\mathrm{PBI}$, the choice of treatment technique has consistently favored external beam PBI, accounting for approximately $70 \%$ of use compared with approximately 25\% MammoSite use, with the remaining cases using traditional catheter brachytherapy. ${ }^{2}$

The Milan Cancer Institute also designed a prospective phase III trial comparing WBRT with intraoperative PBI, delivered with a specially designed mobile linear accelerator using electrons, or the ELIOT technique. The dose is a single dose of 21 Gy in the experimental arm delivered at the time of breast surgery. The standard treatment arm includes postoperative WBRT to a dose of 50 Gy over 5 weeks, followed by a boost dose to the lumpectomy bed. This study accrued 1305 women between November 2000 and December 2007. Results were reported in May 2012 at the Barcelona ESTRO meeting. The local failure rate at 5 years in the WBRT arm was $0.7 \%$, compared with $5.3 \%$ in the PBI intraoperative radiation therapy $\operatorname{arm}(P=.0002)$. Fat necrosis was noted in $16 \%$ of the women assigned to the PBI arm, and those patients also were observed to have an increase in the rate of axillary relapse when compared with the women in the WBRT group. The Milan group announced a future plan for the ELIOT technique, limiting its use to those women older than 60 years, with ER-positive, Tl luminal-type lesions. They also will dose escalate to 22 to $24 \mathrm{~Gy}^{3}$

A third large randomized trial, TARGIT-A, completed accrual and presented results at ASCO in 2010. With a similar design to the Milan trial, women were also randomized to receive either standard WBRT or intraoperative radiation. However, the delivery system in this trial is unique and unusual; the dose in the PBI arm is delivered through a low-energy, $50-\mathrm{kV}$ beam device called the Intrabeam (Carl Zeiss, Germany). The radiation dose is calculated for 20 Gy at the surface of the applicator, but only approximately 5 to $6 \mathrm{~Gy}$ at a distance of $1 \mathrm{~cm}$ into the breast tissue, a reference point commonly accepted in virtually all other PBI studies for dose calculations. ${ }^{4}$

Although the above characteristics of the Intrabeam device allow it to be used in operating rooms without any special shielding, they also raise questions about its efficacy for breast cancer. The authors of the study concluded in part that their results "challenge the dogma that the traditional radiation dose (much higher than targeted intraoperative radiotherapy) is essential for effective tumour control." 3 So what are some of the important details of this study, in terms of understanding its results? First, although the trial was open to women aged 45 years and older, the study population had a median age of 63 years. Among the patients, $86 \%$ had T1 tumors, and more than $90 \%$ had an ER-positive cancer, with $87 \%$ being HER2/ neu-negative. Although not stated in the text, Table 6 in the publication states that the median follow-up time of the group was just 25 months. ${ }^{4}$

Perhaps more interesting is the language in the Intrabeam protocol allowing any women randomized to the PBI trial arm to also receive WBRT for a pathologic finding of "adverse features," such as high-grade disease or lymphovascular invasion. Of the women on the PBI arm, 14\% were actually treated with this additional WBRT. At 4 years, results in terms of local failure in the breast were as follows: 6 patients $(1.2 \%)$ in the PBI group and $5(0.95 \%)$ in the standard WBRT arm. Although this new delivery system and low dose is hypothesis-generating, the conclusion of the authors, which is that for selected patients "a single dose of radiotherapy delivered at the time of surgery should be considered as an alternative to external beam radiotherapy," may be premature. ${ }^{4}$ The study population in this trial is very "good risk," with two-thirds also receiving systemic hormone therapy. With such a short median followup time and the use of the additional WBRT in 14\% of the PBI study arm, the low rate of local failure is not unexpected, and additional analysis with more follow-up seems prudent. ${ }^{4}$

\section{The ASTRO Consensus Guidelines}

To address the gap of missing knowledge from the time of closure of the PBI trials to the time when follow-up is sufficient to analyze the studies for local control and other end points, ASTRO published a consensus statement in 2009. The recommendations for the use of PBI based on current knowledge divide potential patients into "suitable," "cautionary," and "unsuitable" groups.

The main characteristics of the "suitable" group include age of 60 years or older and T1,NO unicen- 
tric ER-positive disease excised with margins of at least $2 \mathrm{~mm}$. Histology should be invasive ductal carcinoma, or one of the favorable subtypes. The cautionary group includes those women aged 50 to 59 years, with a primary tumor between 2.1 and 3 $\mathrm{cm}$, including those with ductal carcinoma in situ and invasive lobular histology; women with closer margins, ER-negative receptor status, and/or limited lymphovascular invasion or a limited extensive intraductal component present are also in this "cautionary" group. The "unsuitable" group includes women under age 50 years, those carrying a BRCA mutation, those requiring neoadjuvant chemotherapy, and those with adverse pathology factors, including node-positive disease, larger primary lesions, positive margins, and multicentric carcinoma. ${ }^{5}$

As a reviewer of the ASTRO consensus product before it was finalized and sent to press, I found the recommendations appropriate given the levels of evidence that the consensus panel had to work with. One of the goals of the panel was to provide clinical guidelines for the general use of PBI until more definitive results from the trials discussed earlier become available, and I believe this was accomplished. Although the manuscript from the Milan-based intraoperative radiation trial is not yet available, the future strategy for the ELIOT technique seems to confirm this ASTRO consensus.

\section{Toxicity Associated With PBI}

Although some of the rationale behind the use of PBI includes the smaller treatment volumes used with the potential for lower exit doses of radiation to nearby critical structures, several reports of unexpected toxicity have been published from single institutions. These reports include a finding of postradiation pneumonitis; Recht et $\mathrm{al}^{6}$ found an incidence of $17 \%$ in their dose-escalation trial, at a total PBI dose of 36 Gy. Their analysis related this side effect to the volume of ipsilateral lung receiving an exit dose of 20 Gy or higher; modification of the PBI treatment plan seems to have resolved this issue.

Hepel et al, ${ }^{7}$ analyzing their patients in the national NSABP/RTOG PBI trial, found a $25 \%$ incidence of grade 2 to 4 subcutaneous fibrosis, with a median follow-up of 15 months. They were able to relate this increase to breast dose-volume parameters, and still reported overall good cosmesis.
Jagsi et $\mathrm{al}^{8}$ from the University of Michigan reported several cases of "unacceptable cosmesis" from a PBI study using similar external beam doses and parameters as the NSABP/RTOG study, but also with active breathing control. With 7 of 34 cases in their study found to have some combination of "volume loss, retraction, contour defect, and telangiectasia," the authors also related these findings to the volume of breast tissue receiving $50 \%$ and $100 \%$ of the prescription dose.

Although these toxicity reports represent findings from just a few of the many centers using some form of PBI as treatment for women with breast cancer, they are a reminder that as any new technique becomes more widely adopted, there is a learning curve for the physician. In the NSABP B-39/RTOG 0413 phase III trial, test cases were required for every center and for each technique of PBI used before the center could enter an actual patient in the study. Rapid review of treatment plans of the first 5 cases each center entered was also performed online by one of the study's principal investigators. Similar unexpected toxicity has not been observed to date. ${ }^{2}$

\section{Hypofractionated WBRT}

In the world of radiation biology, the term alpha-betaratio describes the relationship between response of different tissues, both normal and cancerous, and radiation fraction size. For many years, this ratio for breast cancer was thought to be well above 7 , similar to squamous cell carcinoma. However, experimental data in the late 1980s suggested the alpha-beta ratio for adenocarcinoma is much lower than previously estimated, leading to the design of clinical trials testing this hypothesis.

Specifically, Yarnold et $\mathrm{al}^{9}$ in the United Kingdom designed a trial comparing the standard WBRT dose of 50 Gy in 25 fractions with a 39- or 42.9-Gy total dose delivered in just 13 fractions, on alternate days over 5 weeks. The clinical results confirmed the hypothesis regarding the lower alpha-beta ratio for normal breast tissue and for breast cancer cells, and led to several large trials in the United Kingdom, called the Standardization of Breast Radiotherapy (START) A and B trials, and to a similar trial in Canada. ${ }^{9-12}$

To summarize the results of the 3 English trials, 2236 women were randomized in the START A trial, 2215 in the START B trial, and 1410 in the pilot trial. Results were initially reported with an average 
of 5 years of follow-up, and to date the hypofractionated treatment seems equivalent to the more traditional 25-fraction course in terms of all end points. Whelan et $\mathrm{a}^{12}$ conducted a similar trial in Canada, with an updated report issued in 2011 that included 10 years of follow-up. In the Canadian study, 1233 women received either 50 Gy in 25 fractions over 5 weeks or 42.5 Gy in 16 fractions over 22 days. Again, results were equivalent between the regimens regarding tumor control, side effects, and late effects.

Physicians at Memorial Sloan-Kettering Cancer Center have been using the hypofractionated regimen introduced by Whelan et $\mathrm{a}^{12}$ for almost 10 years. Most of these women are treated in the prone position to avoid exit doses to the lungs and heart, and a simplified form of intensity-modulated radiation is used to ensure a homogenous dose throughout the breast, with the larger daily fraction size. Results are similar to those reported in the trials discussed earlier, including no increase in toxicity associated with the use of a boost dose of radiation when indicated and after systemic chemotherapy, including with adriamycin and/or the taxanes. ${ }^{13}$

\section{Conclusions}

Large prospective randomized PBI trials have accrued well in recent years, and the body of evidence comparing this technique with the standard of care, WBRT, is beginning to be reported, with mixed results. The Milan-based intraoperative radiation study favors the use of WBRT; the Intrabeam trial results, although equivocal with limited follow-up, have many radiation biology issues as discussed. The NSABP B-39/RTOG 0413 trial will reach planned accrual next year, but analysis of first results will be several years in the future. ASTRO has published a thoughtful guide for PBI use until more information from these studies is presented and published.

Results of hypofractionated WBRT trials are available and mature, with more than 7000 women followed out for more than 10 years, and the use of this more convenient and abbreviated form of WBRT seems to be well tolerated by those who meet the trial criteria. Until more evidence is available in favor of the use of PBI, offering women who require WBRT this shorter but equally effective radiation schedule seems to be a reasonable and more convenient option than the traditional 5-week course.

\section{References}

1. McCormick B. Partial-breast radiation for early staged breast cancers: hypothesis, existing data and a planned phase III trial. J Natl Compr Canc Netw 2005;3:301-307.

2. White J. Update on breast cancer open protocols, RTOG Breast Committee. RTOG Semiannual Meeting; June 14-17, 2012; Philadelphia, PA.

3. Orecchia R. Results of the Milan PBI ELIOT Trial. World Congress of Brachytherapy; May 10-12, 2012; Barcelona, Spain.

4. Vaidya J, Joseph D, Tobias J, et al. Targeted intraoperative radiotherapy versus whole breast radiotherapy for breast cancer (TARGIT A Trial): an international, prospective, randomized, non-inferiority phase 3 trial. Lancet 2010;376:91-102. Erratum in: Lancet 2010;376:90.

5. Smith BD, Arthur DW, Buchholz TA, et al. Accelerated partial breast irradiation consensus statement from the American Society for Radiation Oncology (ASTRO). Am Coll Surg 2009;209:269277.

6. Recht A, Ancukiewicz M, Alm El-Din MA, et al. Lung dosevolume parameters and the risk of pneumonitis for patients treated with accelerated partial-breast irradiation using three-dimensional conformal radiotherapy. J Clin Oncol 2009;27:3887-3893.

7. Hepel T, Tokita M, MacAusland SG, et al. Toxicity of threedimensional conformal radiotherapy for accelerated partial breast irradiation. Int J Radiat Oncol Biol Phys 2009;75:1290-1296.

8. Jagsi R, Ben-David MA, Moran JM, et al. Unacceptable cosmesis in a protocol investigating intensity- modulated radiotherapy with active breathing control for accelerated partial-breast irradiation. Int J Radiat Oncol Biol Phys 2010;76:71-78.

9. Yarnold J, Ashton A, Bliss J, et al. Fractionation sensitivity and dose response of late adverse effects in the breast after radiotherapy for early breast cancer: long-term results of a randomized trial Radiother Oncol 2005;75:9-17.

10. Bentzen SM, Agrawal RK, Aird EG, et al. START Trialists' Group. The UK Standardisation of Breast Radiotherapy (START) trial A of radiotherapy hypofractionation for treatment of early breast cancer: a randomised trial. Lancet Oncol 2008;9:331-341.

11. The START Trialists' Group. The UK Standardisation of Breast Radiotherapy (START) Trial B of radiotherapy hypofractionation for treatment of early breast cancer: a randomized trial. Lancet 2008;371:1098-1107.

12. Whelan TJ, Pignol JP, Levine MN, et al. Long-term results of hypofractionated radiation therapy for breast cancer. N Engl J Med 2010;362:513-520.

13. Croog VJ, Wu AJ, McCormick E, Beal KP. Accelerated whole breast irradiation with intensity modulated radiotherapy to the prone breast. lnt J Radiat Oncol Biol Phys 2009;73:88-93. 